\title{
BALIKCI Anna, Lamas, Shamans and Ancestors. Village Religion in Sikkim
}

Brill's Tibetan Studies Library, Leiden-Boston, Brill, 2008, 403 pages

Anne de Sales

\section{(2) OpenEdition}

Journals

Édition électronique

URL : https://journals.openedition.org/emscat/1637

DOI : 10.4000/emscat. 1637

ISSN : 2101-0013

Éditeur

Centre d'Etudes Mongoles \& Sibériennes / École Pratique des Hautes Études

Référence électronique

Anne de Sales, «BALIKCI Anna, Lamas, Shamans and Ancestors. Village Religion in Sikkim », Études mongoles et sibériennes, centrasiatiques et tibétaines [En ligne], 40 | 2009, mis en ligne le 30 décembre 2009, consulté le 13 juillet 2021. URL : http://journals.openedition.org/emscat/1637 ; DOI : https:// doi.org/10.4000/emscat.1637

Ce document a été généré automatiquement le 13 juillet 2021.

(c) Tous droits réservés 


\title{
BALIKCI Anna, Lamas, Shamans and Ancestors. Village Religion in Sikkim
}

\author{
Brill's Tibetan Studies Library, Leiden-Boston, Brill, 2008, 403 pages
}

\author{
Anne de Sales
}

\section{RÉFÉRENCE}

BALIKCI Anna, Lamas, Shamans and Ancestors. Village Religion in Sikkim, Brill's Tibetan Studies Library, Brill (Leiden-Boston, 2008), 403 p.

1 Les relations que les grandes religions entretiennent avec les pratiques religieuses locales constituent un des domaines privilégiés de la recherche en anthropologie pour de bonnes raisons: il faut en effet prendre en compte des données non seulement d'ordre religieux mais aussi historique, économique, sociologique et politique afin de saisir ces phénomènes complexes et l'anthropologue s'est fait une spécialité de combiner ces différentes approches, souvent grâce à une longue expérience de terrain. L'ouvrage d'Anna Balikci offre un exemple particulièrement réussi de ce que cette tradition peut offrir. Lamas, Shamans and Ancestors. Village Religion in Sikkim se présente comme la monographie d'une communauté bouddhiste de l'Himalaya, qui a façonné au cours de son histoire et selon des modalités différentes, une religion de village dans laquelle une vision chamanique $\mathrm{du}$ monde persiste, de façon relativement indépendante, à côté de la religion bouddhique.

2 Le royaume bouddhique du Sikkim est fondé en 1642 par des lamas Nyingma - la plus ancienne des traditions du bouddhisme tibétain. Ce petit pays montagneux est alors peuplé par les Bhotia ou Lhopa, venus du Tibet à une date plus ancienne, et les Lepcha, une population chamaniste indigène, qui a jusqu'ici retenu l'attention des ethnologues beaucoup plus que ne l'ont fait les anciens immigrants tibétains. Si le bouddhisme s'efforça bien d'absorber le culte des ancêtres et des divinités locales, ces rituels n'en ont pas moins formé le cœur de cérémonies exécutées à la fois par les lamas de village et les spécialistes religieux locaux, ici appelés chamanes, qui se rattachent à la religion 
bon - la religion autochtone tibétaine, reconstruite a posteriori, qui a précédé le bouddhisme. L'auteur propose que ces différents spécialistes partagent une même conception de la réalité, selon laquelle le corps des individus (et du coup la santé et la maladie), le territoire, la société et le surnaturel ne sont pas des domaines de la vie sociale indépendants les uns des autres, mais au contraire en perpétuelle interaction. Cette vision du monde, qualifiée par Balikci de chamanique, est à la base d'une division du travail rituel entre les spécialistes religieux qui coexistent sans conflit : aux lamas reviennent plus particulièrement les rituels funéraires tandis que les chamanes sont consultés au contraire pour repousser l'échéance finale.

3 La première partie du livre introduit le lecteur à l'histoire politique et religieuse du Sikkim, et à la "religion de village » et ses acteurs : d'une part le lama de village, qui pratique un bouddhisme non dogmatique et d'autre part le chamane clanique (pawo) et le spécialiste des divinités locales (bongthing), Les deux parties suivantes présentent les contextes dans lesquels ces spécialistes religieux interagissent au sein d'un village en particulier, Tingchim, sur lequel l'auteur centre sa monographie. Ainsi la deuxième partie développe les relations des villageois à leur terre, sur le plan de l'économie agricole et des rites. La troisième partie est centrée sur les rites domestiques qui assurent fertilité et prospérité ; la maisonnée se révèle alors fournir le cadre dans lequel les membres réitèrent leur appartenance à la communauté et la vision chamanique du monde se maintient vivante. La dernière partie se concentre sur le rôle du bouddhisme conventionnel dans les relations que le village entretient avec l'État et avec le monde extérieur. S'il y avait conflit ce serait entre les représentants de ce bouddhisme conventionnel et les partisans d'un bouddhisme villageois plus pragmatique, non pas entre les lamas de village et les chamanes.

Ce qui est particulièrement intéressant dans cette étude est qu'elle permet de comprendre comment différents éléments de la vie sociale convergent pour produire une certaine configuration religieuse à une période donnée puis se modifient pour en produire une autre à une autre période, tout en conservant certains traits caractéristiques. Ainsi la persistance d'une vision chamanique du monde vient en partie de ce que le Sikkim, depuis sa fondation au XVII siècle et jusqu'à son intégration à l'Union Indienne en 1975, était un état peu centralisé avec une royauté faible, incapable d'imposer de façon stricte la religion bouddhique à ses sujets. Les monastères de moines célibataires, qui formaient au Tibet central un soutien politique d'envergure à la monarchie bouddhique, n'ont jamais pu se maintenir au Sikkim pour des raisons en partie démographiques : la culture sur brûlis nécessitait une nombreuse main d'œuvre et une famille pouvait difficilement se priver de l'un de ses fils pour l'envoyer au monastère. Aussi, à Tingchim, la vie rituelle est-elle restée entièrement entre les mains de spécialistes rituels bon jusqu'en 1910, quand pour la première fois un lama est venu s'installer au village. Vers la fin du XIX ${ }^{e}$ siècle, l'introduction d'une culture de rapport, la cardamome, associée à l'immigration d'ouvriers agricoles venus du Népal, a bouleversé cette situation. L'argent et le temps gagnés ont permis aux Lhopo, seuls propriétaires des terres, d'investir dans des formes de culte à la fois plus coûteuses et plus prestigieuses et le village se mit à " produire des lamas au même rythme que la cardamome ». Dans les années 1930 l'influence des lamas s'étendit des cérémonies funéraires aux rites communaux, les chamanes conservant cependant le monopole des rites de cure jusqu'à la mort du dernier d'entre eux à Tingchim, en 1997. 
Contrairement à l'ancienne culture sur brûlis autour de laquelle était structurée une vie sociale faite de relations d'échanges au sein de la communauté des Lhopo, la culture de la cardamome est dépendante des travailleurs népalais et des marchands indiens. Les relations économiques qui fournissaient la trame du tissu social n'ayant plus de raison d'être, c'est sur la sphère rituelle que repose à présent l'unité de la communauté, tout particulièrement les rituels associés au territoire (comme ceux qui président aux récoltes) et au cycle de vie de la maisonnée : de la participation à ces rites, souvent coûteux, dépend l'appartenance des membres à leur communauté. En outre les rites domestiques réaffirment la vision chamanique du monde, en faisant intervenir comme partenaires surnaturels primordiaux les dieux ancestraux et des divinités du pays. Si la fin de la royauté et l'intégration à l'union indienne en 1975 signent la fin du bouddhisme d'État, on assiste cependant depuis les années 90 à un retour de cette religion servie par l'engouement international qu'elle suscite, couplé à une attention croissante portée aux sites sacrés de la nation. Aussi est-ce à présent dans ce souci pour l'environnement et dans les rituels domestiques que survit le fond chamanique étroitement associé, selon l'auteur, à l'identité sikkimaise.

6 Dans la mesure où les domaines de la vie sociale se répondent ainsi les uns aux autres et se transforment du même pas, l'auteur aurait pu y voir l'esquisse d'un système, mais un système ouvert aux innovations. Elle a préféré se centrer sur les débats anthropologiques concernant l'interface entre chamanisme et bouddhisme. L'intérêt de ce travail réside plus encore, nous semble-t-il, dans la présentation d'une étude de cas particulièrement bien documentée sur les transformations sociales et religieuses d'un village sikkimais au $\mathrm{xx}^{\mathrm{e}}$ siècle, ce que le titre ne laisse pas deviner, mais que la lecture de l'ouvrage révèle clairement. Le plan choisi pour exposer cette réalité complexe entraîne d'assez nombreuses répétitions, mais le matériel ethnographique est exceptionnellement riche, varié, et bien décrit. Il ouvre en outre de nombreuses voies comparatives avec d'autres communautés himalayennes. Le livre d'Anna Balikci est une lecture indispensable pour qui s'intéresse à cette région. 